\title{
Regulation of the Nijmegen breakage syndrome 1 gene NBS1 by c-myc, p53 and coactivators mediates estrogen protection from DNA damage in breast cancer cells
}

\author{
ROWENA WAN, JIANCHUN WU, KAITRIN K. BALOUE and DAVID L. CROWE \\ University of Illinois Cancer Center, Chicago, IL 60612, USA
}

Received October 12, 2012; Accepted December 4, 2012

DOI: $10.3892 /$ ijo.2012.1757

\begin{abstract}
In mammalian cells more than $90 \%$ of doublestrand breaks are repaired by NHEJ. Impairment of this pathway is associated with cell cycle arrest, cell death, genomic instability and cancer. Human diseases such as Nijmegen breakage syndrome, due to mutations in the NBS1 gene, produce defects in resection of double-strand breaks. NBS1 hypomorphic mutant mice are viable, and cells from these mice are defective in $\mathrm{S}$ phase and G2/M checkpoints. NBS1 polymorphisms have been associated with increased risk of breast cancer. We previously demonstrated that estradiol protected estrogen receptor (ER)-positive (+) breast cancer cell lines against double-strand breaks and cell death. We now demonstrate that protection from double-strand break damage in $\mathrm{ER}^{+}$cells is mediated via regulation by c-myc, p53, CBP and SRC1 coactivators in intron 1 of the NBS1 gene. We concluded that NBS1 is responsible for estradiol-mediated protection from double-strand breaks in $\mathrm{ER}^{+}$breast cancer cells.
\end{abstract}

\section{Introduction}

Double-strand break repair is mediated by two major repair pathways, homologous recombination (HR) or non-homologous end joining (NHEJ; reviewed in ref. 1). In mammalian cells more than $90 \%$ of double-strand breaks are repaired by NHEJ. Impairment of either pathway is associated with cell cycle arrest, cell death, genomic instability and cancer (2). Human diseases such as Nijmegen breakage syndrome (NBS) due to mutations in the NBS1 gene result in defects in resection of double-strand breaks (3). NBS1 functions as part

Correspondence to: Professor David L. Crowe, University of Illinois Cancer Center, 801 S. Paulina Street, Room 530C, Chicago, IL 60612, USA

E-mail: dlcrowe@uic.edu

Key words: Rad50, Mre11, MMTV-neu, p53, transcription factor of the Mre11/Rad50/NBS1 (MRN) complex whose functions are not restricted to HR but are also involved NHEJ (4).

NBS is a rare human autosomal recessive disorder caused by hypomorphic mutations. This disorder is characterized by growth retardation, immunodeficiency, microcephaly and cancer predisposition. At the cellular level, NBS is characterized by radiosensitivity, chromosomal breakage and defective cell cycle checkpoints. NBS1 null mutations result in early embryonic lethality (5), but NBS1 hypomorphic mutants are viable (6). Cells from these mice are defective in $S$ phase and G2/M checkpoints. Heterozygous mice with an NBS1 null mutation in addition to homozygous animals with hypomorphic mutations are predisposed to cancer. Conditional NBS1 mutant mice have been characterized (7). For example, neuronal inactivation of NBS1 results in chromosomal breaks, microcephaly, growth retardation, cerebellar defects and ataxia. The MRN complex is essential for maintaining genomic integrity, cell viability and checkpoint activation.

MRN polymorphisms have been associated with increased risk of breast cancer (8-10). MRN expression was reduced in the majority of breast tumors (11). Low expression of MRN correlated with increased histologic grade and estrogen receptor negativity. Response to radiotherapy correlated with high expression of the MRN complex. Patients with high numbers of ionizing radiation induced NBS1 foci had aggressive breast cancer phenotypes $(12,13)$.

Estradiol has been shown to markedly enhance proliferation of mammary gland epithelium and estrogen receptor (ER) $\alpha$ positive (+) breast cancer cells (14). ER is a member of a large family of ligand dependent transcription factors that include steroid, retinoid, thyroid and vitamin D receptors. ER have functional domains for DNA binding, ligand binding, dimerization, and transcriptional activation. Nuclear receptors such as ER require coactivator proteins such as CREB binding protein $(\mathrm{CBP})$ and steroid receptor coactivator 1 (SRC1) to activate target gene transcription (15). We previously demonstrated that estradiol protected $\mathrm{ER}^{+}$breast cancer cell lines against double-strand breaks and cell death (16). Ectopic ER expression was sufficient to produce these effects and this protection involved the coactivator CBP. We now demonstrate that this protection from double-strand break damage is mediated via regulation by c-myc, p53 and coactivators in intron 1 of the NBS1 gene. 


\section{Materials and methods}

Cell culture and stable transfection. The human mammary epithelial and breast cancer cell lines used in this study were purchased from the American Type Culture Collection and cultured in Dulbecco's modified Eagle's medium without phenol red, $10 \%$ charcoal-resin treated fetal bovine serum, and $40 \mu \mathrm{g} / \mathrm{ml}$ gentamicin in a humidified atmosphere of $5 \% \mathrm{CO}_{2}$ at $37^{\circ} \mathrm{C}$. Cultures were treated with $100 \mathrm{nM} \mathrm{E} 2$ for $4 \mathrm{~h}, 3 \mathrm{~Gy}$ ionizing radiation, combined $\mathrm{E} 2$ and radiation or vehicle. For some experiments, cells were transfected with $2 \mu \mathrm{g}$ c-myc, CBP, SRC1 or neomycin resistance plasmid using Lipofectamine according to manufacturer's recommendations (Invitrogen, Carlsbad, CA). Cells were selected in $400 \mu \mathrm{g} / \mathrm{ml} \mathrm{G} 418$ for 14 days. Resistant clones were picked for expansion and characterization. For inhibition of gene expression experiments, cells were transfected with siRNA to ER $\alpha$, Mre11, Rad50, NBS1 or control siRNA according to manufacturer's protocol (Dharmacon, Lafayette, $\mathrm{CO}$ ).

DNA damage and apoptosis analysis. DNA damage was quantitated by single cell gel electrophoresis. Treated cells were mixed with $0.5 \%$ low melting point agarose and added to microscope slides coated with $1.5 \%$ agarose. Cells were alkali denatured ( $\mathrm{pH} 13.0)$, subjected to electrophoresis at $0.86 \mathrm{~V} / \mathrm{cm}$ for $25 \mathrm{~min}$ and stained with ethidium bromide. The tail moment (DNA migration $x$ tail intensity) of 50 randomly selected cells was analyzed from each slide using imaging software. For apoptosis assays, human mammary epithelial and breast cancer cell cultures were fixed with $70 \%$ ethanol at $-20^{\circ} \mathrm{C}$ for $30 \mathrm{~min}$ and washed with PBS. Cultures of mouse mammary epithelial cells were incubated with terminal deoxynucleotidyl transferase and fluorescein conjugated dUTP at $37^{\circ} \mathrm{C}$ for $30 \mathrm{~min}$ followed by washing in PBS. The percentage of apoptotic cells was determined by flow cytometry.

Western blot analysis. Protein was extracted in 1X Laemmli buffer from treated human mammary epithelial and breast cancer cell lines. Total cellular protein $(75 \mu \mathrm{g})$ was separated by SDS-PAGE on $10 \%$ resolving gels under denaturing and reducing conditions. Separated proteins were electroblotted to PVDF membranes according to manufacturer's recommendations (Roche Applied Science, Indianapolis, IN). Blots were incubated with antibodies to ER $\alpha$, Mre11, Rad50, NBS1, c-myc, CBP, SRC1 or $\beta$-actin for $16 \mathrm{~h}$ at $4^{\circ} \mathrm{C}$. After washing in Tris buffered saline containing $0.1 \%$ Tween-20 (TBST, $\mathrm{pH}$ 7.4), blots were incubated for $30 \mathrm{~min}$ at room temperature with anti-IgG secondary antibody conjugated to horseradish peroxidase. Following extensive washing in TBST, bands were visualized by the enhanced chemiluminescence method (Roche Applied Science). Bands were quantitated by laser densitometry.

Chromatin immunoprecipitation. Treated human mammary epithelial or breast cancer cells were fixed in $1 \%$ formaldehyde for $10 \mathrm{~min}$ at room temperature. Cells were washed in PBS and lysed in immunoprecipitation buffer containing protease inhibitors for $30 \mathrm{~min}$ at $4^{\circ} \mathrm{C}$, sheared and centrifuged at $10,000 \mathrm{x} \mathrm{g}$ for $10 \mathrm{~min}$. Supernatants were cleared with $2 \mu \mathrm{g}$ sheared salmon sperm DNA, $20 \mu \mathrm{l}$ preimmune serum, and $20 \mu \mathrm{l}$ protein A/G sepharose beads for $2 \mathrm{~h}$ at $4^{\circ} \mathrm{C}$. Aliquots of the supernatant were used as input DNA for normalization. Immunoprecipitation using anti-myc, -p53, -CBP, -SRC1 or -acetylated histone H3 antibodies (Santa Cruz Biotechnology, Santa Cruz, CA) was performed overnight at $4^{\circ} \mathrm{C}$. Preimmune IgG was used as the negative control antibody. Immunoprecipitates were washed extensively in immunoprecipitation buffer, resuspended in $10 \mathrm{mM}$ Tris-HCl, 1 mM EDTA (TE, pH 8.0) and incubated at $65^{\circ} \mathrm{C}$ for $6 \mathrm{~h}$ to reverse crosslinks. The supernatants were extracted with phenol/chloroform and ethanol precipitated. Following washing in $70 \%$ ethanol, pellets were dried and suspended in $50 \mu \mathrm{l}$ TE. For real-time PCR, $1 \mu \mathrm{l}$ of template was amplified in buffer containing $10 \mathrm{mM}$ Tris- $\mathrm{HCl}(\mathrm{pH} 8.3), 50 \mathrm{mM}$ $\mathrm{KCl}, 2.5 \mathrm{mM} \mathrm{MgCl}, 200 \mathrm{nM}$ each dNTP and $100 \mathrm{ng}$ each primer (5'-GATAACCCTTTCCCACTGATTG-3' and 5'-GAGA ACTGCTTGAACCCAG-3') flanking the myc and p53 binding sites in the NBS1 first intron (accession AY566246; 3024-3029 bp and 3124-3134 bp, respectively). The optimized cycle parameters were one cycle at $94^{\circ} \mathrm{C}$ for $3 \mathrm{~min}$ followed by 25 cycles of $94^{\circ} \mathrm{C}$ for $25 \mathrm{sec}, 58^{\circ} \mathrm{C}$ for $60 \mathrm{sec}$, $72^{\circ} \mathrm{C}$ for $60 \mathrm{sec}$ and one final cycle at $72^{\circ} \mathrm{C}$ for $10 \mathrm{~min}$ (iCycler, Bio-Rad).

Nucleosomal mapping. Nuclei were isolated from parental human breast cancer cell lines treated with E2, IR, E2+IR, or vehicle. Chromatin was digested to mononucleosomal form with micrococcal nuclease (Roche Applied Science). The digestion was stopped by addition of $50 \mathrm{mM}$ EDTA. Nuclei were lysed in $1 \%$ SDS and treated with $0.1 \mathrm{mg}$ proteinase $\mathrm{K}$ overnight at $37^{\circ} \mathrm{C}$. DNA was purified by phenol/chloroform extraction and ethanol precipitation. DNA was suspended in TE buffer and analyzed by agarose gel electrophoresis to ensure digestion to mononucleosomal fragments. These fragments were eluted from the gel and used as PCR templates to determine nucleosomal occupancy of NBS1 intron 1 . Undigested genomic DNA was used as the positive control and template free samples were used as the negative control.

Transient transfection, double-strand break repair and NBS1 intron 1 analysis. pACT-luc luciferase vector was digested with $\mathrm{Xba \textrm {I }}$ and $B s t \mathrm{EII}$ or EcoRV and $\mathrm{XhoI}$ restriction enzymes and purified by gel electrophoresis followed by end-filling with Klenow fragment of DNA polymerase (17). A total of $1 \mu \mathrm{g}$ of each linear plasmid was transiently transfected into triplicate cultures of 50\% confluent human breast cancer cell lines using Lipofectamine according to the manufacturer's recommendations (Invitrogen). The 728 bp homology between the two plasmids can reconstitute luciferase activity which correlates with DNA double-strand break repair activity. Undigested pACT-luc vector was used as the positive control and $1 \mu \mathrm{g} \beta$-galactosidase expression plasmid was used to normalize for transfection efficiency. In separate experiments, triplicate cultures of $50 \%$ confluent cells were transiently transfected with $2 \mu \mathrm{g}$ of pGL3 luciferase reporter vector containing 5 ' flanking constructs of the NBS1 promoter, exon 1 and intron $1(-360 /+1076)$, lacking the promoter $(-17 /+1076)$ or lacking intron 1 $(-360 /+88)$. Intron 1 was cloned into pGL3 vector and transiently transfected with $1 \mu \mathrm{g}$ c-myc, p53, CBP, SRC1 

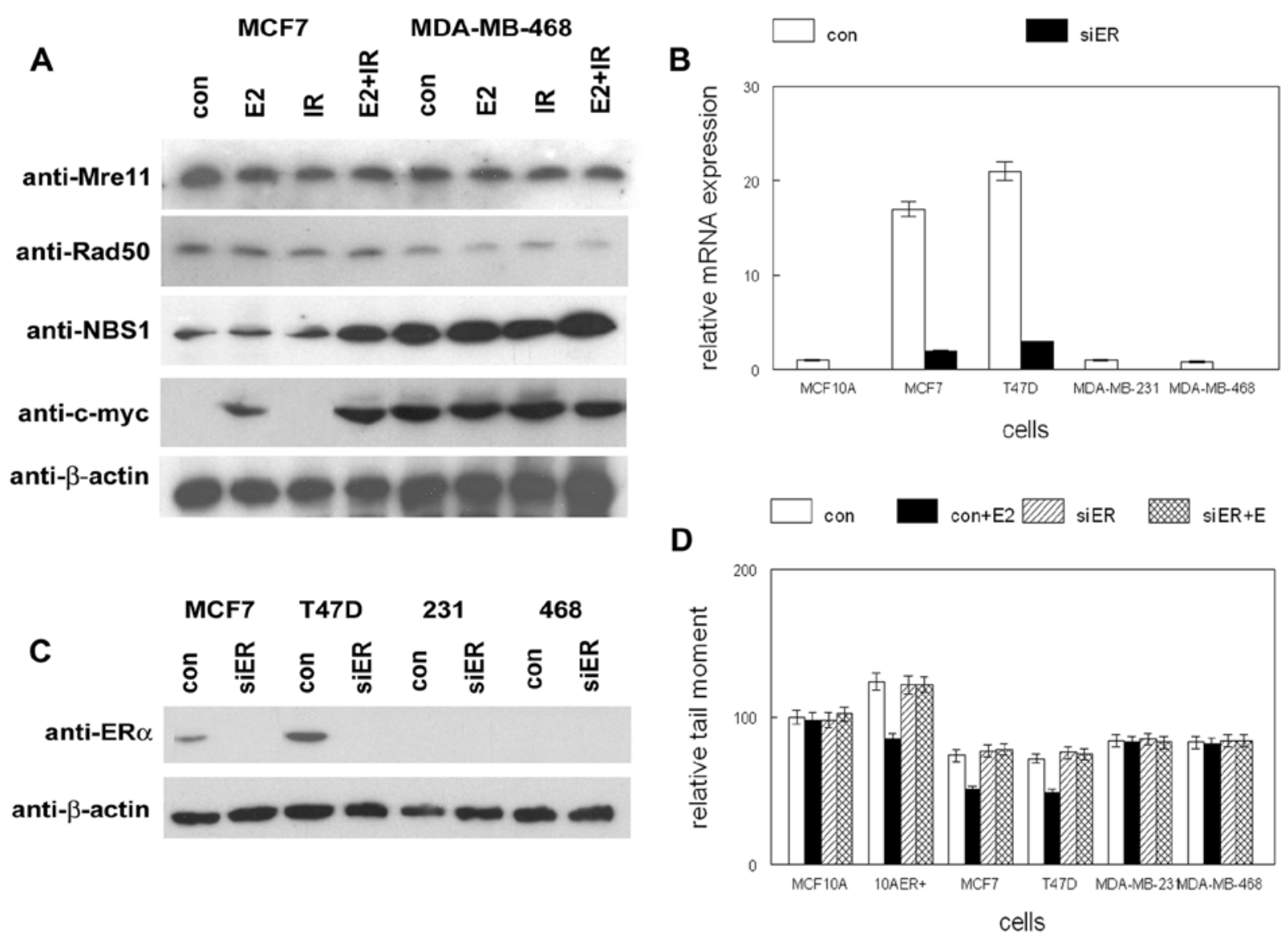

E

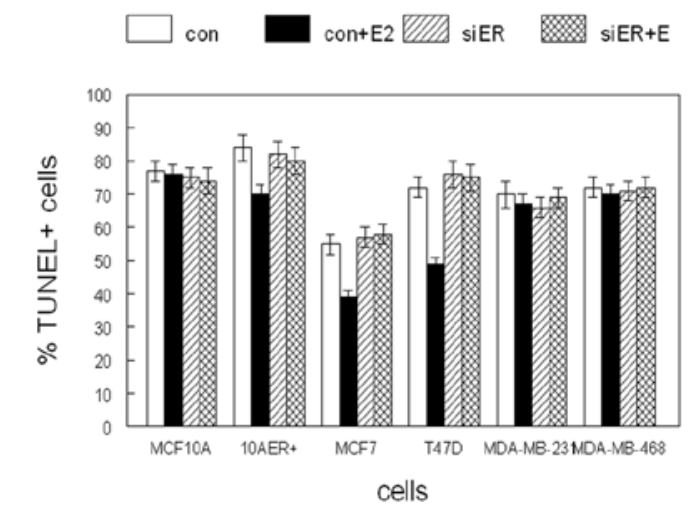

Figure 1. The combination of estradiol and ionizing radiation induces NBS1 expression in $\mathrm{ER}^{+}$human breast cancer cell lines. (A) The ER ${ }^{+}$breast cancer cell line MCF7 and ER line MDA-MB-468 were treated with estradiol (E2), ionizing radiation (IR), combination (E2+IR) or vehicle (con). Protein extracts from these cells were subjected to western blot analysis using antibodies indicated at left. (B) Human breast cancer cell lines were transfected with ER or control (con) siRNA followed by qRT-PCR. (C) Human breast cancer cell lines were transfected with ER or control (con) siRNA followed by western blot analysis using antibodies indicated at left. (D) E2 protection from ionizing radiation induced DNA damage is dependent on ER expression. The immortalized human breast epithelial line MCF10A, MCF10A stably expressing ER (10AER ${ }^{+}$), and human breast cancer cell lines were transfected with ER siRNA (siER) or control siRNA (con), treated with E2 (+E2) or vehicle, and exposed to ionizing radiation. Relative tail moment is shown. (E) E2 protection from ionizing radiation induced apoptosis is dependent on ER expression. MCF10A, MCF10A stably expressing ER (10AER ${ }^{+}$) and human breast cancer cell lines were transfected with ER siRNA (siER) or control siRNA (con), treated with E2 (+E2) or vehicle, and exposed to ionizing radiation. Percent TUNEL positive cells is shown. Error bars represent SEM of three independent experiments.

or blank expression plasmids. Separate cultures were transfected with pGL3 vector containing point mutations in the intron 1 myc (CACcaGC) or p53 (GGGgccGCTCC) binding sites. Cultures were treated with $\mathrm{E} 2$, ionizing radiation or vehicle for $24 \mathrm{~h}$. Cells were harvested and reporter gene activity determined using a commercially available kit and luminometer (Applied Biosystems, Carlsbad, CA). Luciferase activity was normalized to $\beta$-galactosidase levels for each sample. Statistical significance was determined by ANOVA.

\section{Results}

We previously determined that estradiol (E2) treatment decreased DNA damage and increased survival of $\mathrm{ER}^{+}$human breast cancer cell lines exposed to ionizing radiation (IR) (16). To determine the mechanism of this protection, we treated $\mathrm{ER}^{+}$and $\mathrm{ER}^{-}$human breast cancer cell lines with E2, IR or E2 followed by IR. As shown in Fig. 1A, NBS1 protein expression was induced by 5 -fold in $\mathrm{ER}^{+} \mathrm{MCF} 7$ cells when treated 
A

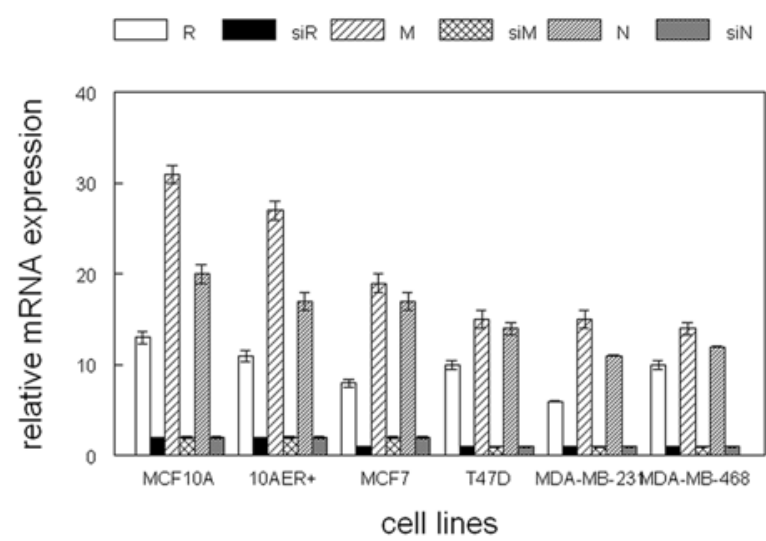

C

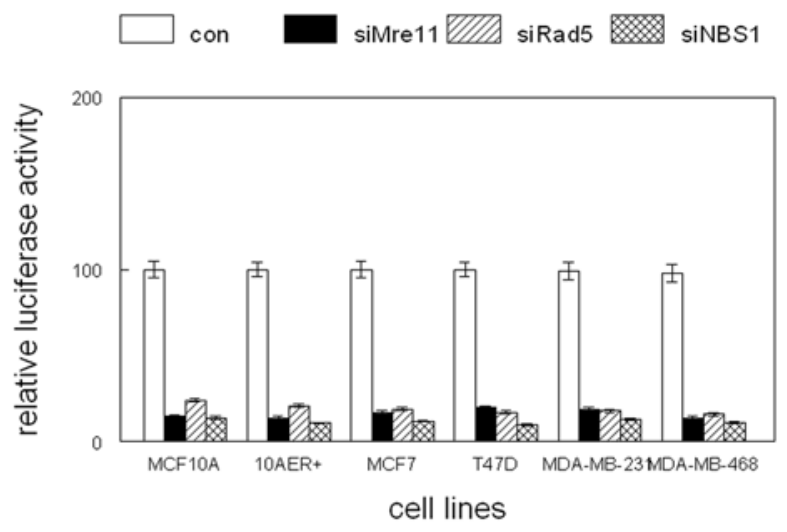

E

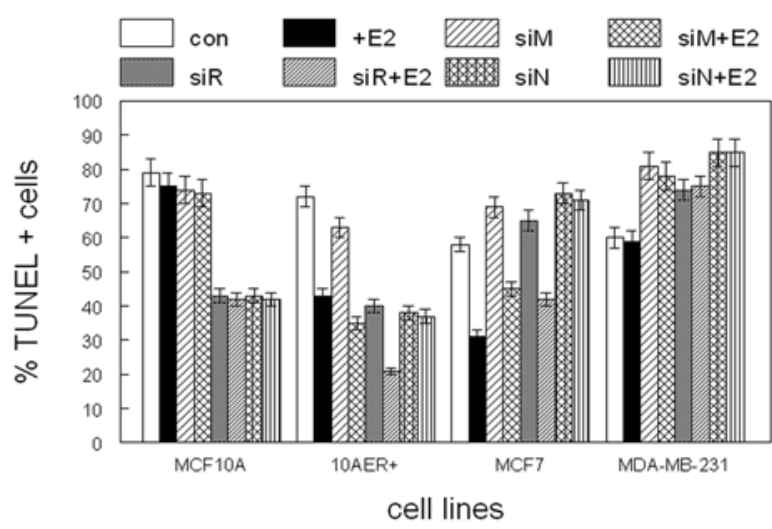

with E2 followed by IR. No NBS1 expression changes were observed in ER- MDA-MB-468 cells. No changes in expression of other MRN gene products (Mre11, Rad50) in response to E2 or IR were observed. Similar results were observed in $\mathrm{ER}^{+}$T47D and ER ${ }^{-}$MDA-MB-231 cells (data not shown).
B

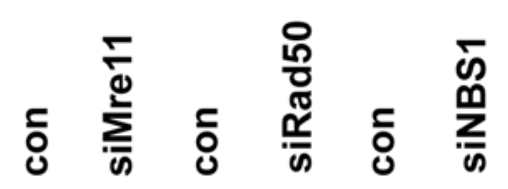

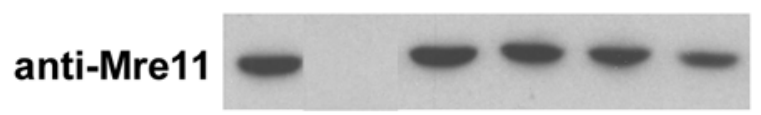

anti-Rad50

anti-NBS1

anti- $\beta$-actin
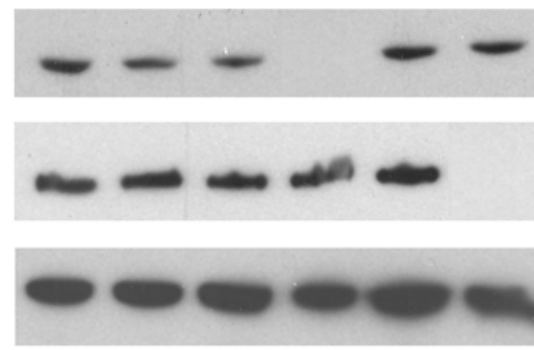

D

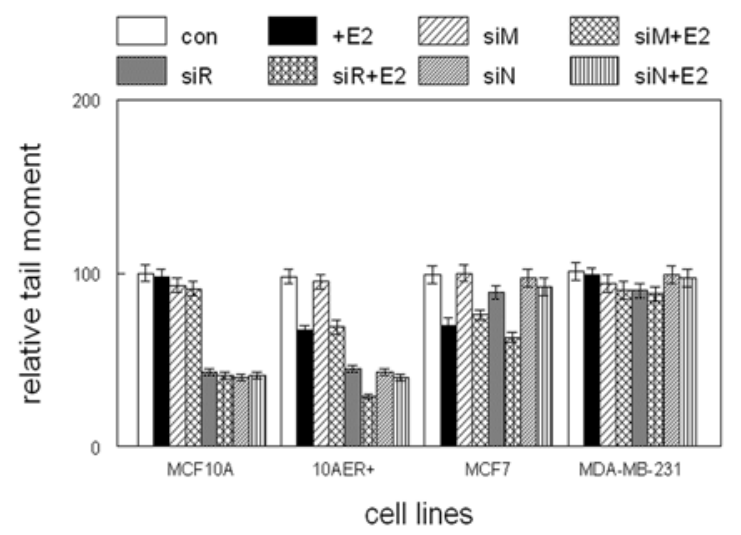

Figure 2. siRNA inhibits expression of MRN gene products. (A) MCF10A, MCF10A stably expressing ER (10AER $)$, and human breast cancer cell lines were transfected with siRNA to Mre11 (siM), Rad50 (siR), NBS1 (siN) or control siRNAs (M, R, N) followed by qRT-PCR. (B) MCF10A, MCF10A stably expressing ER $\left(10 \mathrm{AER}^{+}\right)$, and human breast cancer cell lines were transfected with siRNA to Mre11 (siMre11), Rad50 (siRad50), NBS1 (siNBS1) or control siRNA (con) followed by western blot analysis with antibodies indicated at left. (C) siRNAs to MRN gene products inhibits double-strand break repair. MCF10A, MCF10A stably expressing ER (10AER ${ }^{+}$, and human breast cancer cell lines were transfected with siRNA to Mre11 (siMre11), Rad50 (siRad50), NBS1 (siNBS1) or control siRNA (con) and digested luciferase reporter vector. (D) NBS1 mediates E2 protection from IR mediated DNA damage. MCF10A, MCF10A stably expressing ER (10AER $)$, and human breast cancer cell lines were transfected with siRNA to Mre11 (siM), Rad50 (siR), NBS1 (siN) or control siRNA (con), treated with E2 (+E2) or vehicle, and exposed to ionizing radiation. Relative tail moment is shown. (E) NBS1 mediates E2 protection from IR mediated apoptotic cell death. MCF10A, MCF10A stably expressing ER $\left(10 \mathrm{AER}^{+}\right)$, and human breast cancer cell lines were transfected with siRNA to Mre11 (siM), Rad50 (siR), NBS1 (siN) or control siRNA (con), treated with E2 (+E2) or vehicle, and exposed to ionizing radiation. Percent of apoptotic cells is shown. Error bars indicate SEM of three independent experiments.

These results indicate that both E2 and IR were required to induce NBS1 expression in $\mathrm{ER}^{+}$breast cancer cell lines.

To determine if $\mathrm{E} 2$ mediated protection from double-strand break damage was dependent on ER, we transfected $\mathrm{ER}^{+}$and $\mathrm{ER}^{-}$human mammary epithelial and breast 
A

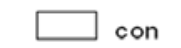

ש+E2

एखि IR

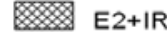

을

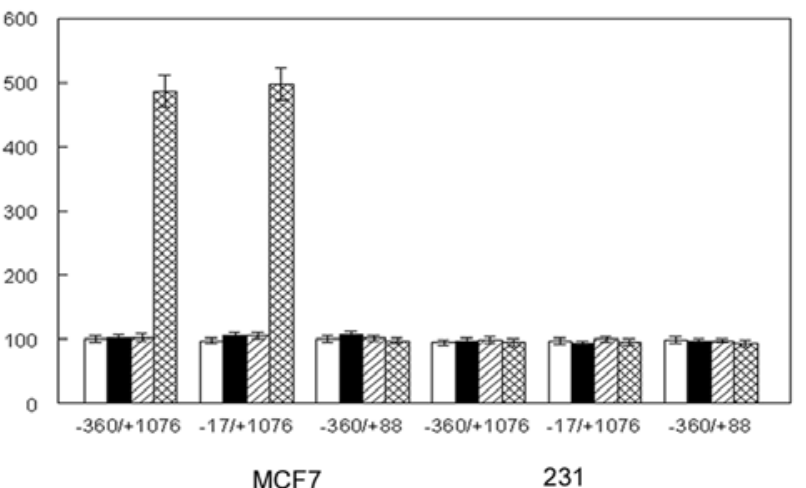

B

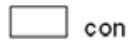

+E2

VII IR E2+IR
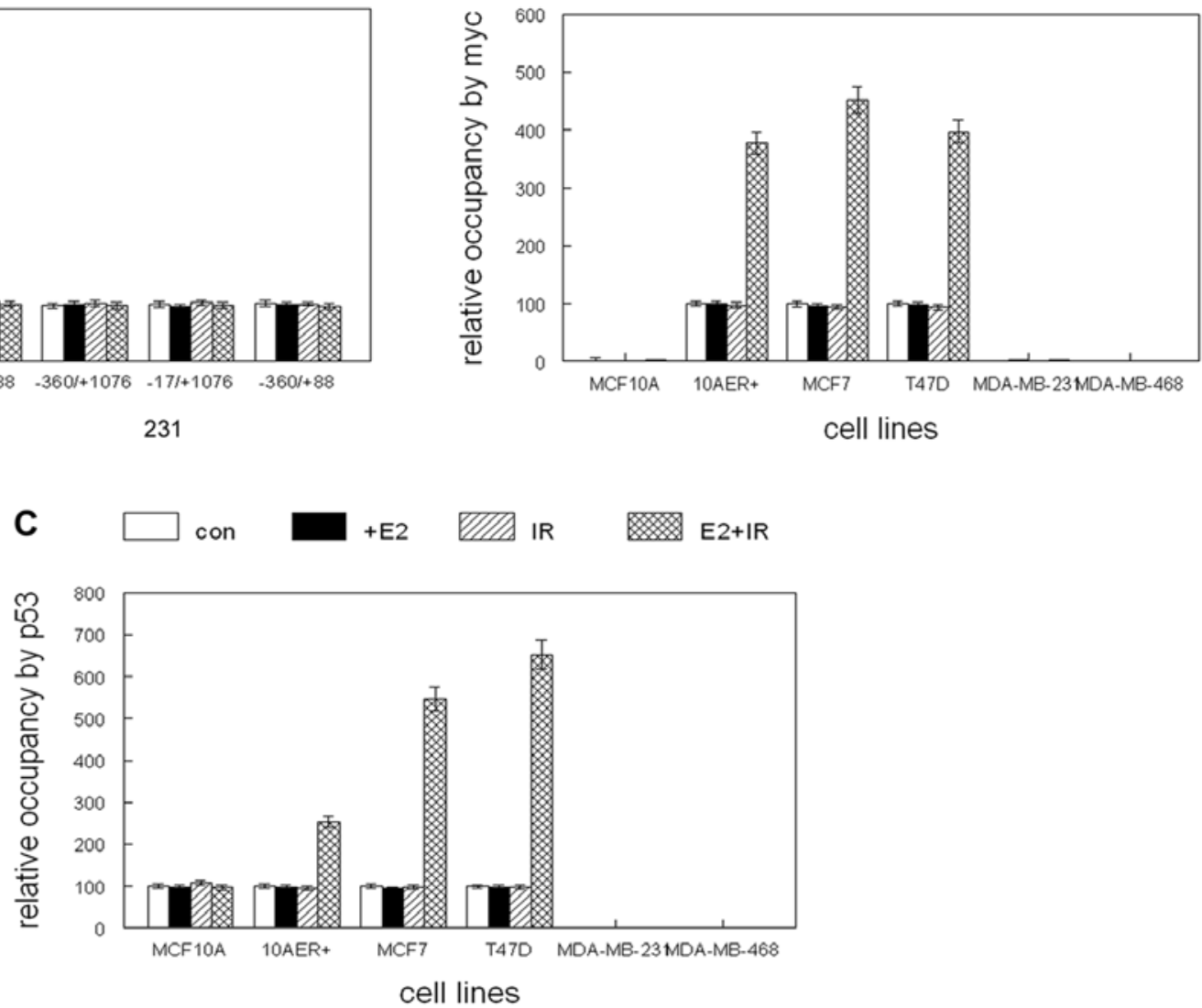

Figure 3. (A) Intron 1 mediates E2 and IR induction of NBS1 gene expression. Constructs containing the NBS1 promoter, exon 1 and intron 1 (-360/+1076), lacking the promoter $(-17 /+1076)$ or lacking intron $1(-360 /+88)$ were fused to the luciferase reporter and transiently transfected into MCF7 or MDA-MB-231 cells. (B) c-myc occupancy of NBS1 intron 1 is induced by the combination of E2 and ionizing radiation in $\mathrm{ER}^{+}$cells. MCF10A, MCF10A stably expressing ER $\left(10 \mathrm{AER}^{+}\right)$, and human breast cancer cell lines were treated with E2 (+E2), ionizing radiation (IR), combined E2 and IR (E2+IR), or vehicle (con) and subjected to chromatin immunoprecipitation. Relative occupancy of intron 1 by c-myc is shown. (C) p53 occupancy of NBS1 intron 1 is induced by the combination of E2 and ionizing radiation in $\mathrm{ER}^{+}$cells. MCF10A, MCF10A stably expressing ER $\left(10 \mathrm{AER}^{+}\right)$, and human breast cancer cell lines were treated with E2 (+E2), ionizing radiation (IR), combined E2 and IR (E2+IR), or vehicle (con) and subjected to chromatin immunoprecipitation. Relative occupancy of intron 1 by p53 is shown.

cancer cell lines with siRNA to ER. Expression of ER mRNA and protein following siRNA transfection is shown in Fig. 1B and C. ER expression was reduced in $\mathrm{ER}^{+} \mathrm{MCF} 7$ and T47D cells by $>90 \%$ following siRNA transfection. Treatment of $\mathrm{ER}^{+}$cells with $\mathrm{E} 2$ reduced double-strand break damage by $25-30 \%$ following IR as determined by tail moment $(\mathrm{p}<0.04$; Fig. 1D). This protective effect was abolished by ER siRNA transfection and was not observed in ER cells. Treatment of $\mathrm{ER}^{+}$cells with E2 reduced apoptosis by similar magnitude as determined by TUNEL assay ( $<<0.03$; Fig. 1E). This effect was completely inhibited by ER siRNA transfection and was not observed in ER cells. These results indicate that the protective effect of E2 against double-strand break damage was mediated by ER.

Pretreatment with E2 prior to ionizing radiation induced NBS1 expression in $\mathrm{ER}^{+}$human mammary epithelial and breast cancer cells (Fig. 1). To determine the role of the MRN complex in mediating this response, we transfected these cells with siRNAs to Mre11, Rad50 or NBS1. Expression of these gene products following siRNA transfection is shown in Fig. 2A and B. Expression of Mre11, Rad50 or NBS1 was reduced by $90 \%$ in siRNA transfected cultures. Transfection of siRNAs to Mre11, Rad50, or NBS1 inhibited recombination of luciferase plasmids by $70-80 \%$ ( $<<0.002$; Fig. 2C), providing functional confirmation of reduced expression of these gene products. To determine the effects of MRN gene product inhibition, we exposed siRNA transfected cells to E2 or vehicle followed by IR. As shown in Fig. 2D, NBS1 siRNA blocked the protective effects of E2 against double-strand break damage. These effects were observed only in $\mathrm{ER}^{+}$ cells. Similar effects of NBS1 siRNA were observed on E2 mediated protection against ionizing radiation induced apoptosis (Fig. 2E). These results indicate that NBS1 mediates the $\mathrm{E} 2$ protective effects against ionizing radiation induced double-strand break damage and apoptosis.

To determine if NBS1 induction was mediated by transcription, we transiently transfected a reporter construct containing the $5^{\prime}$ flanking, exon 1 and intron 1 regions of the gene into human breast cancer cell lines prior to E2 and IR treatment. As shown in Fig. 3A, the combination of E2 and IR induced reporter activity by 5 -fold in ER ${ }^{+}$MCF7 cells $(\mathrm{p}<0.01)$. Deletion of the proximal promoter region had no effect on reporter activity, but removal of intron 1 completely abolished luciferase expression. These effects were not 


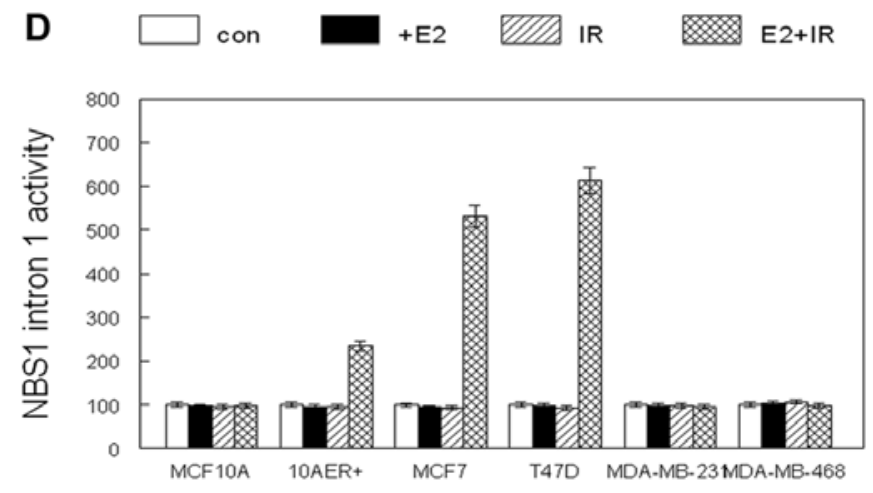

cell lines

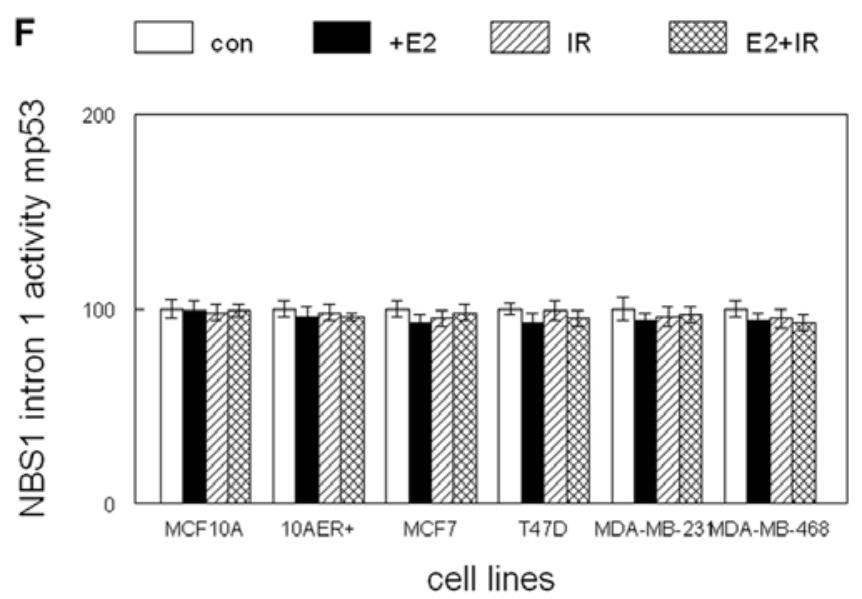

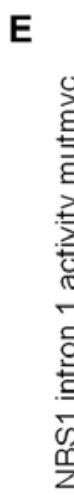

G
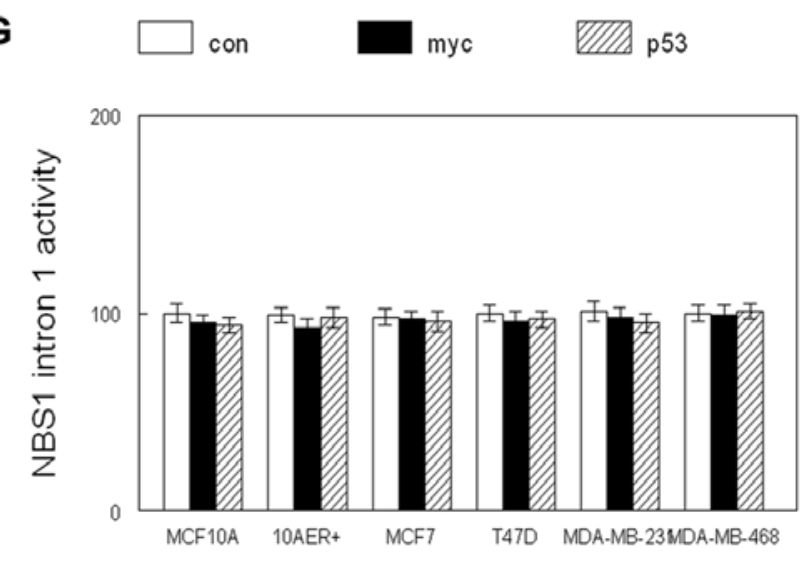

cell lines

Figure 3. Continued. (D) NBS1 intron activity is induced by the combination of E2 and ionizing radiation in $\mathrm{ER}^{+}$cells. MCF10A, MCF10A stably expressing ER $\left(10 \mathrm{AER}^{+}\right)$, and human breast cancer cell lines were transfected with the luciferase reporter construct and treated with E2 (+E2), ionizing radiation (IR), combined E2 and IR (E2+IR) or vehicle (con). Relative luciferase activity is shown. (E) Mutation of the E box site in the NBS1 intron 1 inhibits induction of activity by the combination of E2 and IR. MCF10A, MCF10A stably expressing ER $\left(10 \mathrm{AER}^{+}\right)$, and human breast cancer cell lines were transfected with the luciferase reporter construct containing a mutation in the E box site and treated with E2 (+E2), ionizing radiation (IR), combined E2 and IR (E2+IR) or vehicle (con). Relative luciferase activity is shown. (F) Mutation of the p53 binding site in the NBS1 intron 1 inhibits induction of activity by the combination of E2 and IR. MCF10A, MCF10A stably expressing ER $\left(10 \mathrm{AER}^{+}\right)$, and human breast cancer cell lines were transfected with luciferase reporter construct containing a mutation in the p53 binding site and treated with E2 (+E2), ionizing radiation (IR), combined E2 and IR (E2+IR) or vehicle (con). Relative luciferase activity is shown. (G) c-myc and p53 individually fail to activate NBS1 intron 1. MCF10A, MCF10A stably expressing ER (10AER $)$, and human breast cancer cell lines were transfected with the luciferase reporter construct and expression vectors for c-myc, p53 or control vector (con). Error bars indicate SEM of three independent experiments.

observed in ER- MDA-MB-231 cells. In silico analysis of potential transcription factor binding sites in NBS1 intron 1 mediating E2 and DNA damage responses revealed a myc and p53 site in close proximity. To determine relative occupancy of the myc and p53 binding sites in the NBS1 intron 1 in response to E2 and IR we performed chromatin immunoprecipitation. As shown in Fig. 3B, the combination of E2 and IR enhanced binding of c-myc to this region of NBS1 intron 1 by 4 -fold in $\mathrm{ER}^{+}$human breast epithelial cell lines $(p<0.02)$. E2 or IR alone had no effect on $c$-myc binding to the NBS1 intron 1. ER cells showed no binding of c-myc to the NBS1 intron 1. Similarly the combination of E2 and IR induced $\mathrm{p} 53$ binding to this region by 4-7-fold in $\mathrm{ER}^{+}$ cells ( $p<0.01$; Fig. 3C). E2 or IR alone had no effect on p53 binding to NBS1 intron 1. ER cells showed no binding of p53 to the NBS1 intron 1. NBS1 intron 1 activity was strongly induced by the combination of E2 and IR (2-6-fold in $\mathrm{ER}^{+}$ cells; p $<0.05$; Fig. 3D). E2 or IR alone had no effect on NBS1 intron 1 activity and no induction was observed in ER cells. Mutation of the myc or p53 binding sites abolished the inductive effects of E2 and IR on NBS1 intron 1 activity (Fig. 3E and F). Transient overexpression of c-myc or p53 alone failed to activate NBS1 intron 1 activity (Fig. 3G). These results indicate that E2 and IR were required to activate the NBS1 intron 1 via cooperative c-myc and p53 binding to their cognate binding sites.

We previously determined that the protective effects of E2 on IR induced DNA damage was dependent on the epigenetic coactivator CBP (16). To determine if the coactivators CBP and SRC1 were recruited to the myc and p53 binding sites of the NBS1 intron 1, we performed chromatin immunoprecipitation. As shown in Fig. 4A and B, the combination of $\mathrm{E} 2$ and IR recruited $\mathrm{CBP}$ and $\mathrm{SRC} 1$ to this region of intron 1 in $\mathrm{ER}^{+}$cells (2-3-fold increased occupancy; $\left.\mathrm{p}<0.04\right)$. Corresponding acetylation of histone $\mathrm{H} 3$ in this region was increased by 2-3-fold which reduced nucleosomal occu- 
A

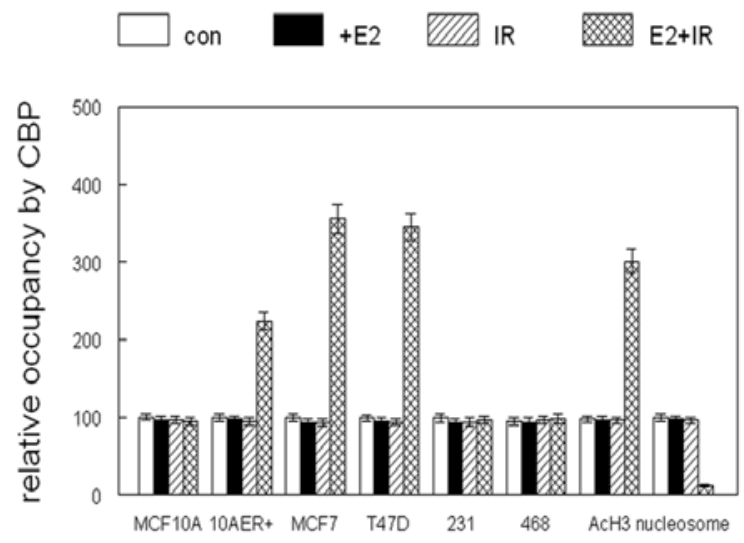

C

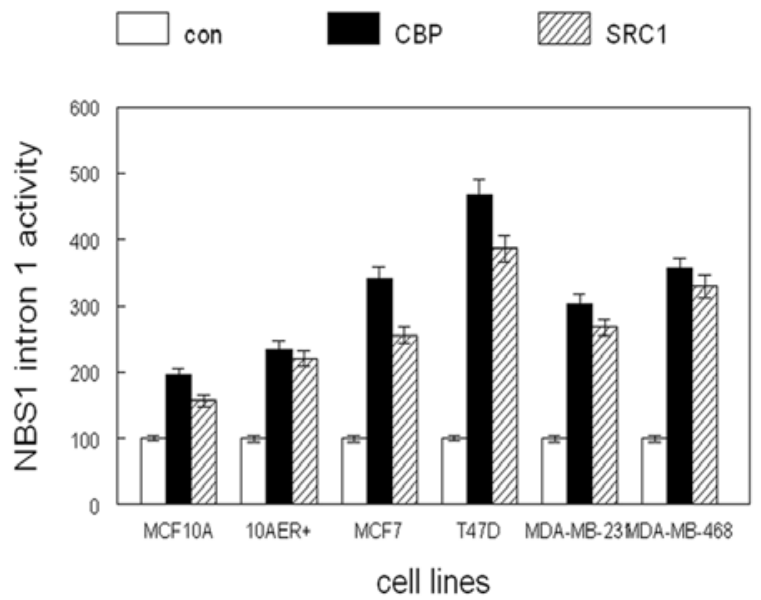

B

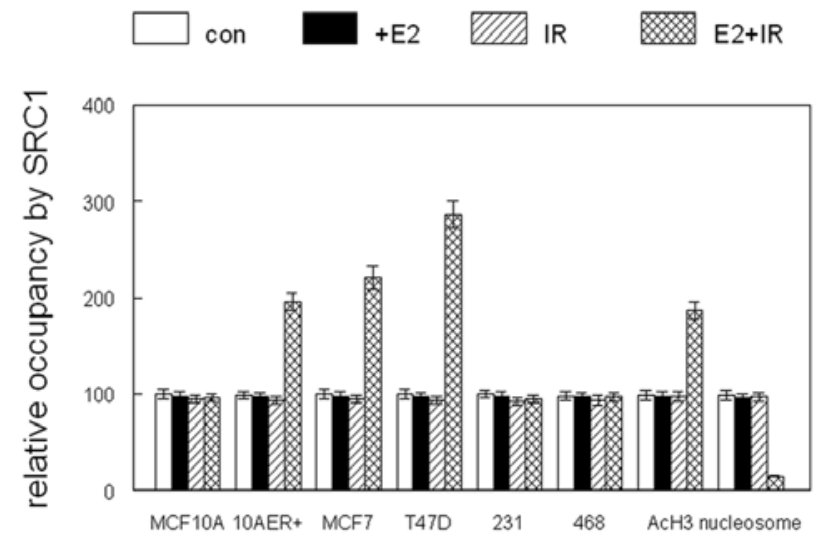

D

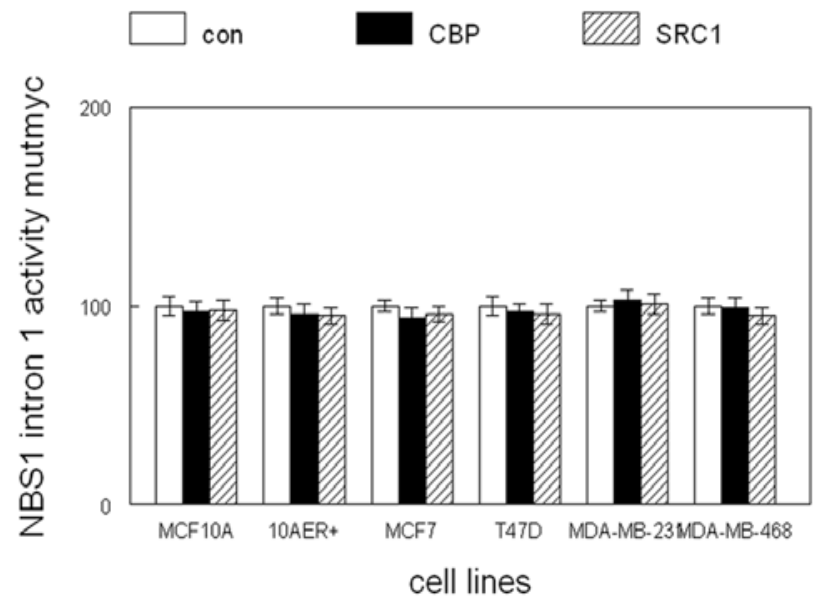

Figure 4. CBP and SRC1 coactivators are recruited to the NBS1 intron 1 by the combination of E2 and ionizing radiation. (A) MCF10A, MCF10A stably expressing ER (10AER ${ }^{+}$, and human breast cancer cell lines were treated with E2 (+E2), ionizing radiation (IR), combined E2 and IR (E2+IR) or vehicle (con) and subjected to chromatin immunoprecipitation. Relative occupancy of intron 1 by CBP, AcH3, or nucleosomes is shown. (B) MCF10A, MCF10A stably expressing ER (10AER $)$, and human breast cancer cell lines were treated with E2 (+E2), ionizing radiation (IR), combined E2 and IR (E2+IR) or vehicle (con) and subjected to chromatin immunoprecipitation. Relative occupancy of intron 1 by SRC1, AcH3, or nucleosomes is shown. (C) CBP and SRC1 activate NBS1 intron 1 activity. MCF10A, MCF10A stably expressing ER (10AER $)$ and human breast cancer cell lines were transiently transfected with luciferase reporter construct and expression vectors for CBP, SRC1 or control vector (con). (D) Mutation of the E box site inhibits coactivator mediated induction of NBS1 intron 1 activity. MCF10A, MCF10A stably expressing ER $\left(10 \mathrm{AER}^{+}\right)$and human breast cancer cell lines were transfected with luciferase reporter construct containing a mutation in the E box site and expression vectors for CBP, SRC1 or control vector (con).

pancy by $90 \%$. E2 or IR alone did not increase coactivator occupancy of this region and no increase in CBP or SRC1 binding was observed following E2 and IR treatment of $\mathrm{ER}^{-}$cells. However, transient overexpression of CBP induced NBS1 intron 1 activity by $2-5$-fold and SRC1 overexpression induced intron activity by $2-4$-fold in both $\mathrm{ER}^{+}$and $\mathrm{ER}^{-}$cell lines ( $\mathrm{p}<0.01$; Fig. 4C). Mutation of the myc or p53 binding sites abolished the ability of CBP or SRC1 to induce NBS1 intron 1 activity (Fig. 4D and E). Constitutive overexpression of p53 induced apoptosis in human breast cancer cell lines (data not shown), but stable c-myc expression in combination with IR induced endogenous NBS1 expression by 3-5-fold in $\mathrm{ER}^{+}$cell lines (Fig. 4F). CBP or SRC1 stable overexpression was sufficient to induce NBS1 gene expression by 2-4-fold in $\mathrm{ER}^{+}$human breast cancer cell lines (Fig. 4G). These results indicate that $\mathrm{E} 2$ and IR recruited coactivators to the myc and p53 binding sites of the NBS1 intron 1. Coactivator induction of NBS1 gene expression was dependent on these sites, and c-myc functionally substituted for E2 treatment in $\mathrm{ER}^{+}$cells.
CBP and SRC1 functionally substituted for E2 and IR induction of NBS1 gene expression, indicating that the coactivators were sufficient to reproduce these effects.

\section{Discussion}

Our previously published studies indicated that E2 treatment decreased DNA damage and improved survival of $\mathrm{ER}^{+}$human breast cancer cell lines following IR treatment (16). We now demonstrate that the combination of E2 and IR treatment induces NBS1 expression in $\mathrm{ER}^{+}$but not $\mathrm{ER}^{-}$human breast cancer cell lines. While inhibition of gene products in the MRN complex inhibited DNA repair, NBS1 was responsible for mediating the anti-apoptotic effects of E2 in irradiated $\mathrm{ER}^{+}$breast cancer cell lines. A previous study demonstrated that cells from mice expressing a C-terminal deleted NBS1 exhibited decreased apoptosis (18). Additionally E2 was previously shown to sustain the growth of irradiated breast cancer cell lines (19). This effect was due to inactivation 
E

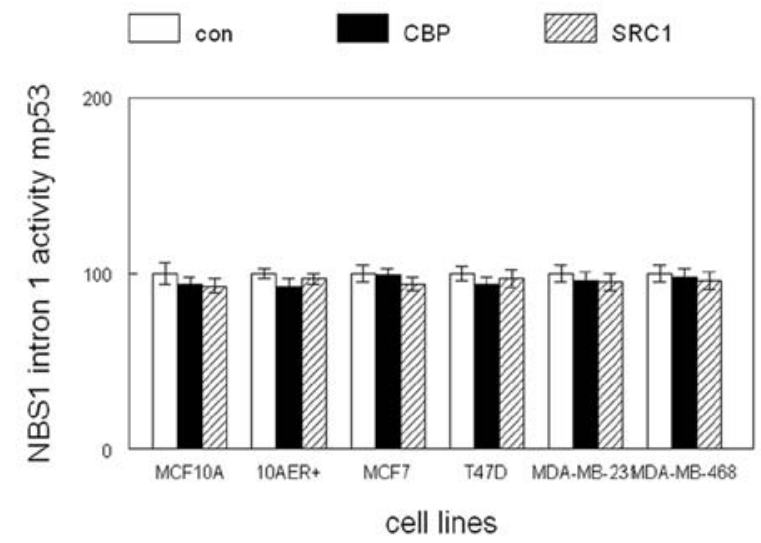

G

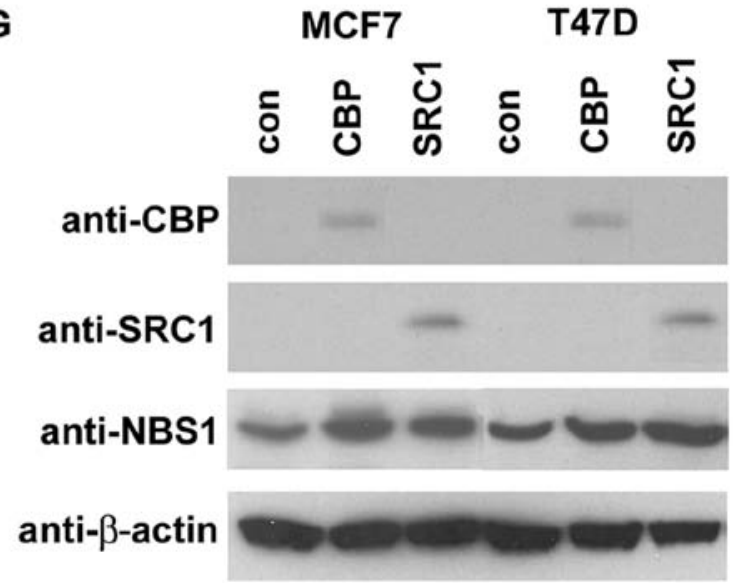

$\mathbf{F}$
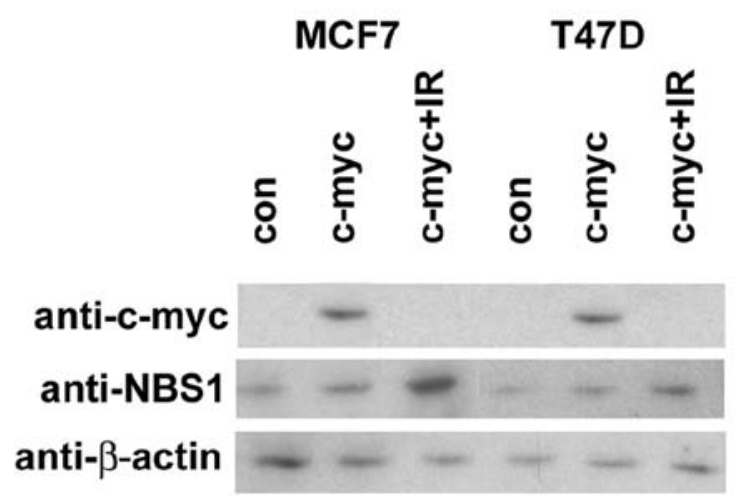

Figure 4. Continued. (E) Mutation of the p53 binding site inhibits coactivator mediated induction of NBS1 intron 1 activity. MCF10A, MCF10A stably expressing ER (10AER $)$, and human breast cancer cell lines were transfected with luciferase reporter construct containing a mutation in the p53 binding site and expression vectors for CBP, SRC1 or control vector (con). (F) Stable overexpression of c-myc substitutes for E2 treatment in IR mediated induction of NBS1 gene expression. The human breast cancer cell lines MCF7 and T47D were stably transfected with c-myc or control (con) expression vector and exposed to ionizing radiation (IR). c-myc, NBS1, and $\beta$-actin expression was determined by western blot analysis. (G) Stable overexpression of CBP or SRC1 substitutes for E2 and IR mediated induction of NBS1 expression. The human breast cancer cell lines MCF7 and T47D were stably transfected with $\mathrm{CBP}, \mathrm{SRC1}$, or control (con) expression vectors. CBP, SRC1, NBS1, and $\beta$-actin expression was determined by western blot analysis.

of p21 which sustained Rb hyperphosphorylation allowing increased cell cycle progression in irradiated cells. These studies demonstrate important control of cell cycle and apoptosis by E2 and NBS1 in human breast cancer cells.

Our results demonstrated that induction of c-myc by E2 and $\mathrm{p} 53$ by IR was required for increased NBS1 expression in $\mathrm{ER}^{+}$human breast cancer cell lines. The lack of NBS1 induction by $\mathrm{E} 2$ or IR alone may be due to the proximity of the myc and p53 response elements in the NBS1 intron 1 (20). p53 has been shown to bind to half sites in target gene promoters (21). Myc or p53 overexpression alone was not sufficient to induce NBS1 expression, but myc expression could substitute for E2 in irradiated cells. A previous report demonstrated that ER could bind directly to p53 and repress the function of the tumor suppressor (22). This interaction may provide an additional mechanism by which activated ER may inactivate p53 to facilitate cell cycle progression and inhibit apoptosis.

Our results demonstrated that $\mathrm{CBP}$ and $\mathrm{SRC} 1$ coactivators were recruited to the myc and p53 response elements in the NBS1 intron 1 and were sufficient to activate gene expression. Previous studies demonstrated that SRC1 physically interacts with p53 and potentiated p53 mediated transactivation (23). The coactivators $\mathrm{CBP} / \mathrm{p} 300$ associate with and acetylate p53, and results in acetylation of histones in p53 target gene promoters (24-26). These studies demonstrate the importance of coactivator function in mediating the effects of DNA damage response in human breast cancer cells.

Mutations in the NBS1 gene have been associated with increased risk of breast cancer $(9,10,27,28)$. Persistent radiation induced NBS1 foci has been associated with chromosomal instability and increased breast cancer risk (13). In mice, NBS1 null mutation is embryonic lethal but heterozygosity renders mice susceptible to tumor formation (5). However, mammary tumors are uncommon in mouse strains with reduced NBS1 function (6). Defects in cellular proliferation were noted in the cells of NBS1 deficient mice in previous studies (7). Loss of p53 has been shown to greatly increase tumorigenesis in NBS1 mutant mice, suggesting that p53 mediated DNA damage response may be responsible for apoptosis and increased tumor latency (29). A previous study demonstrated nuclear export of NBS1 following ionizing radiation as a mechanism of downregulating the DNA damage response (30). Loss of NBS1 has been shown to induce supernumerary centrosomes similar to those observed in BRCA1 deficient cells, leading to increased chromosomal instability (31). These studies demonstrate that impaired NBS1 function can result in cellular proliferation defects leading to increased tumor latency. It is interesting to speculate that tumorigenic clones that escape defective proliferation may be more aggressive and metastatic due to chromosomal aberrations induced by diminished NBS1 function.

\section{Acknowledgements}

This study was supported by Susan G. Komen for the Cure award BCTR0504295 and Department of Defense Breast Cancer Research Program award W81XWH-10-1-0081 to DLC. 


\section{References}

1. Hakem R: DNA damage repair; the good, the bad, and the ugly. EMBO J 27: 589-605, 2008.

2. Niida $\mathrm{H}$ and Nakanishi M: DNA damage checkpoints in mammals. Mutagenesis 21: 3-9, 2006.

3. Thoms KM, Kuschal C and Emmert S: Lessons learned from DNA repair defective syndromes. Exp Dermatol 16: 532-544, 2007.

4. Sung P and Klein H: Mechanism of homologous recombination: mediators and helicases take on regulatory functions. Nature Rev Mol Cell Biol 7: 739-750, 2006.

5. Kang J, Bronson RT and Xu Y: Targeted disruption of NBS1 reveals its roles in mouse development and DNA repair. EMBO J 21: 1447-1455, 2002.

6. Dumon-Jones V, Frappart PO, Tong WM, et al: Nbn heterozygosity renders mice susceptible to tumor formation and ionizing radiation induced tumorigenesis. Cancer Res 63: 7263-7269, 2003.

7. Frappart PO, Tong WM, Demuth I, et al: An essential function for NBS1 in the prevention of ataxia and cerebellar defects. Nat Med 11: 538-544, 2005.

8. Bartkova J, Tommiska J, Oplustilova L, et al: Aberrations of the MRE11-RAD50-NBS1 DNA damage sensor complex in human breast cancer: MRE11 as a candidate familial cancer predisposing gene. Mol Oncol 2: 296-316, 2008.

9. Bogdanova N, Feschchenko S, Schurmann P, et al: Nijmegen breakage syndrome mutations and risk of breast cancer. Int J Cancer 122: 802-806, 2008.

10. Lu J, Wei Q, Bondy ML, et al: Polymorphisms and haplotypes of the NBS1 gene are associated with risk of sporadic breast cancer in non-Hispanic white women less than or equal to 55 years. Carcinogenesis 27: 2209-2216, 2006.

11. Hsu HM, Wang HC, Chen ST, Hsu GC, Shen CY and Yu JC: Breast cancer risk is associated with the genes encoding the DNA double strand break repair Mre11/Rad50/Nbs1 complex. Cancer Epidemiol Biomarkers Prev 16: 2024-2032, 2007.

12. Soderlund K, Stal O, Skoog L, Rutqvist LE, Nordenskjold B and Askmalm MS: Intact Mre11/Rad50/Nbs1 complex predicts good response to radiotherapy in early breast cancer. Int J Rad Oncol Biol Physics 68: 50-58, 2007.

13. Someya M, Sakata K, Tauchi H, Matsumoto Y, Nakamura A, Komatsu $\mathrm{K}$ and Hareyama $\mathrm{M}$ : Association of ionizing radiation induced foci of NBS1 with chromosomal instability and breast cancer susceptibility. Rad Res 166: 575-582, 2006.

14. Russo IH and Russo J: Role of hormones in cancer initiation and progression. J Mammary Gland Biol Neoplasia 3: 49-61, 1998.

15. Goodman RH and Smolik S: CBP/p300 in cell growth, transformation, and development. Genes Dev 14: 1553-1577, 2000.

16. Crowe DL and Lee MK: New role for nuclear hormone receptors and coactivators in regulation of BRCA1 mediated DNA repair in breast cancer cell lines. Breast Cancer Res 8: 1-12, 2006.

17. Eggleston $\mathrm{P}$ and Zhao Y: A sensitive and rapid assay for homologous recombination in mosquito cells: impact of vector topology and implications for gene targeting. BMC Genet 2 : 21-29, 2001.
18. Stracker TH, Morales M, Couto SS, Hussein H and Petrini JHJ: The carboxy terminus of NBS1 is required for induction of apoptosis by the MRE11 complex. Nature 447: 218-222, 2007.

19. Toillon RA, Magne N, Laios I, et al: Estrogens decrease gamma ray induced senescence and maintain cell cycle progression in breast cancer cells independently of p53. Int J Rad Oncol Biol Physics 67: 1187-1200, 2007.

20. Chiang YC, Teng SC, Su YN, Hsieh FJ and Wu KJ: c-myc directly regulates the transcription of the NBS1 gene involved in DNA double strand break repair. J Biol Chem 278: 19286-19291, 2003.

21. Cai BH, Chen JY, Lu MH, Chang LT, Lin HC, Chang YM and Chao CF: Functional four base A/T gap core sequence CATTAG of p53 response elements specifically bound tetrameric p53 differently than two base A/T gap core sequence CATG bound both dimeric and tetrameric p53. Nuc Acids Res 37: 1984-1990, 2009.

22. Liu W, Konduri SD, Bansai S, et al: Estrogen receptor alpha binds p53 tumor suppressor protein directly and represses its function. J Biol Chem 281: 9837-9840, 2006.

23. Lee SK, Kim HJ, Kim JW and Lee JW: Steroid receptor coactivator 1 and its family members differentially regulate transactivation by the tumor suppressor protein p53. Mol Endocrinol 13: 1924-1933, 1999.

24. Barlev NA, Liu L, Chehab NH, Mansfield K, Harris KG, Halazonetis TD and Berger SL: Acetylation of p53 activates transcription through recruitment of coactivators/histone acetyltransferases. Mol Cell 8: 1243-1254, 2001.

25. Mujtaba S, He Y, Zeng L, et al: Structural mechanism of the bromodomain of the coactivator CBP in p53 transcriptional activation. Mol Cell 13: 251-263, 2004.

26. Watts GS, Oshiro MM, Junk DJ, Wozniak RJ, Watterson S, Domann FE and Futscher BW: The acetyltransferase p300/CBP associated factor is a p53 target gene in breast tumor cells. Neoplasia 6: 187-194, 2004

27. Walsh $\mathrm{T}$ and King MC: Ten genes for inherited breast cancer. Cancer Cell 11: 103-105, 2007.

28. Nowak J, Mosor M, Ziolkowska I, et al: Heterozygous carriers of the I171V mutation of the NBS1 gene have a significantly increased risk of solid malignant tumors. Eur J Cancer 44: 627-630, 2008.

29. Kang J, Ferguson D, Song H, Bassing C, Eckersdorff M, Alt FW and $\mathrm{Xu}$ Y: Functional interaction of $\mathrm{H} 2 \mathrm{AX}, \mathrm{NBS} 1$, and p53 in ATM dependent DNA damage responses and tumor suppression. Mol Cell Biol 25: 661-670, 2005.

30. Vissinga CS, Yeo TC, Warren S, Brawley JV, Phillips J, Cerosaletti $K$ and Concannon P: Nuclear export of NBN is required for normal cellular responses to radiation. Mol Cell Biol 29: 1000-1006, 2009.

31. Shimada M, Sagae R, Kobayashi J, Habu T and Komatsu K: Inactivation of the Nijmegen breakage syndrome gene leads to excess centrosome duplication via the ATR/BRCA1 pathway. Cancer Res 69: 1768-1775, 2009. 